\title{
Geochemical processes of unconventional reservoirs genesis in Bazhenov formation source rocks: mineral matrix and organic matter transformations investigation
}

ANTON KALMYKOV, MARGARITA TIKHONOVA, DINA GAFUROVA, NATALIA BALUSHKINA, GEORGY KALMYKOV AND ANDREW BYCHKOV

Lomonosov Moscow State University

Presenting Author: a.g.kalmykov@gmail.com

Shale oil is one of the main alternative carbon-based source to support oil level when "standard" oil reserves are lowering. Shale oil deposits are found all over the world, but differences in deposition time and sedimentary conditions requires different ways for shale oil search and development. Bazhenov formation contains the highest amounts of shale oil in the world. Meanwhile huge area of distribution, variety in mineral composition and organic matter maturity, different types of unconventional reservoirs and their genesis embarrass detection of reservoirs expansion and oil production. Latest investigations show that reservoirs in Bazhenov formation were formed by secondary transformation of both mineral matrix and organic matter, and several conversion processes may play the role. Also certain sedimentary conditions are necessary for reservoirs to be formed. Geochemical investigations would allow to reconstruct paleogeographic conditions, determine the genesis of secondary processes and investigate changes in organic matter.

New complex method of rocks investigations was used. On one sample X-ray phase and X-ray fluorescense analysis, scanning electronic microscopy, inductive coupled plasma massspectrometry, thin sections microscopy combined with pyrolysis, luminescence microscopy, extraction and gas chromatography with mass spectrometry investigations were made. This complex analysis allowed to determined that one of the main reservoirs is formed due to dissolution of radiolaria skeletons and dolomite and quartz growth. Another type of reservoirs is formed due to the thermal flow and kerogen transformation, organic porosity formation. The third type of reservoirs is apatite lenses formed on shelf edges. In all cases organic matter maturation to at least middle of oil window is required.

To model the processes resulting unconventional reservoirs forming experiments in autoclaves under high temperatures with addition of water and reagents were performed. The kinetics of the reservoirs formation were studied to show how changes in mineral matrix and organic matter influence reservoir properties of the rocks. The results provide geochemical basis to create geological model of Bazhenov formation on different areas and increase effectiveness of reservoir search. In addition, heating experiments provide conditions for formation stimulation, that would increase oil production and develop oil of necessary composition. 